\title{
Positive periodic solutions for second order differential equations with impulsive effects
}

\author{
Weibing Wang ${ }^{1 *}$ and Xuxin Yang ${ }^{2}$
}

\section{"Correspondence:}

wwbing2013@126.com

'Department of Mathematics,

Hunan University of Science and

Technology, Xiangtan, Hunan

411201, P.R. China

Full list of author information is

available at the end of the article

\begin{abstract}
The existence of positive periodic solutions for a class of second order impulsive differential equations is studied. By using a fixed point theorem in cone, we obtain two existence results, which extend some known results.
\end{abstract}

MSC: 34B18; 34B37

Keywords: impulsive differential equation; periodic solution; fixed point theorem

\section{Introduction}

In this paper, we discuss the existence of positive periodic solutions for the following second order impulsive differential equation:

$$
\left\{\begin{array}{l}
u^{\prime \prime}(t)+(a-b) u^{\prime}(t)+f(t, u(t))=0, \quad t \neq t_{k}, \\
\Delta u\left(t_{k}\right)=I_{k}\left(u\left(t_{k}\right)\right), \quad \Delta u^{\prime}\left(t_{k}\right)=J_{k}\left(u\left(t_{k}\right)\right), \quad k \in \mathbb{Z},
\end{array}\right.
$$

where $a, b$ are positive constants, $\Delta u\left(t_{k}\right)=u\left(t_{k}^{+}\right)-u\left(t_{k}^{-}\right), \Delta u^{\prime}\left(t_{k}\right)=u^{\prime}\left(t_{k}^{+}\right)-u^{\prime}\left(t_{k}^{-}\right), u\left(t_{k}^{+}\right)$ and $u\left(t_{k}^{-}\right)$represent the right limit and left limit of $u(t)$ at $t_{k}$, respectively, $u^{\prime}\left(t_{k}^{-}\right)=$ $\lim _{h \rightarrow 0^{-}} h^{-1}\left[u\left(t_{k}+h\right)-u\left(t_{k}\right)\right], u^{\prime}\left(t_{k}^{+}\right)=\lim _{h \rightarrow 0^{+}} h^{-1}\left[u\left(t_{k}+h\right)-u\left(t_{k}\right)\right]$.

Throughout this paper, we suppose that the following conditions are fulfilled:

(P) $f(t+T, u)=f(t, u), T>0$ is a constant, $\lim _{k \rightarrow \pm \infty} t_{k}= \pm \infty, t_{k}<t_{k+1}$. There exists $p \in \mathbb{N}$ such that $I_{k+p}(u)=I_{k}(u), J_{k+p}(u)=J_{k}(u), t_{k+p}=t_{k}+T$.

To define the solution of (1.1), we introduce the space $P C^{r}(\mathbb{R})=\left\{u: \mathbb{R} \rightarrow \mathbb{R} \mid u^{(j)}(t)\right.$ is continuous at $t \neq t_{k}$, left continuous at $t=t_{k}$, and each $u^{(j)}\left(t_{k}^{+}\right)$exists for $k \in \mathbb{Z}$, where $j=$ $0,1, \ldots, r\}$.

By a solution of (1.1) we mean a function $x \in P C^{2}(\mathbb{R})$ satisfying (1.1).

The theory of impulsive differential equations has been a significant development in the last two decades. Periodic solutions and periodic boundary value problems of impulsive differential equations have received considerable attention and much literature has been published; for instance, see [1-5] and the references therein. It should be noted that compared to first order impulsive differential equations, there exist very few existence results of positive periodic solutions for second order impulsive equations, especially for second order impulsive equations with derivative term, see [6-11]. In [12], authors considered the

(c) 2015 Wang and Yang. This article is distributed under the terms of the Creative Commons Attribution 4.0 International License (http://creativecommons.org/licenses/by/4.0/), which permits unrestricted use, distribution, and reproduction in any medium, provided you give appropriate credit to the original author(s) and the source, provide a link to the Creative Commons license, and indicate if changes were made. 
special case $a=b$ of (1.1), in which the functions $f(\cdot, u), I_{k}(u), J_{k}(u)$ satisfy the given growth conditions at the origin and infinity. Hence, one cannot obtain the multiplicity of periodic solutions under their conditions. If $0<t_{1}<t_{2}<\cdots<t_{p}<T$, our problem is equivalent to the periodic boundary value problem

$$
\left\{\begin{array}{l}
u^{\prime \prime}(t)+(a-b) u^{\prime}(t)+f(t, u(t))=0, \quad t \neq t_{k}, t \in[0, T] \\
\Delta u\left(t_{k}\right)=I_{k}\left(u\left(t_{k}\right)\right), \quad \Delta u^{\prime}\left(t_{k}\right)=J_{k}\left(u\left(t_{k}\right)\right), \quad k=1,2, \ldots, p, \\
u(0)=u(T), \quad u^{\prime}(0)=u^{\prime}(T) .
\end{array}\right.
$$

In recent paper [13], authors discussed the special case of (1.2)

$$
\left\{\begin{array}{l}
-u^{\prime \prime}(t)+\rho^{2} u(t)=g(t, u(t)), \quad t \neq t_{k}, t \in[0,2 \pi], \\
\Delta u\left(t_{k}\right)=I_{k}\left(u\left(t_{k}\right)\right), \quad \Delta u^{\prime}\left(t_{k}\right)=J_{k}\left(u\left(t_{k}\right)\right), \quad k=1,2, \ldots, p, \\
u(0)=u(2 \pi), \quad u^{\prime}(0)=u^{\prime}(2 \pi)
\end{array}\right.
$$

and obtained multiplicity of positive solutions of (1.3), where they made the best of the properties about the Green's function of

$$
\left\{\begin{array}{l}
-u^{\prime \prime}(t)+\rho^{2} u(t)=0 \\
u(0)=u(2 \pi), \quad u^{\prime}(0)=u^{\prime}(2 \pi)
\end{array}\right.
$$

However, their method is invalid for (1.1).

In this paper, by using a fixed point theorem in cone, we obtain two existence theorems of a single positive periodic solution for (1.1) under suitable behavior of functions $f, I_{k}, J_{k}$ on some closed set. In addition, some information on the location of periodic solution is obtained, which can lead to the results on multiple periodic solutions.

\section{Main results}

Put $u=x, u^{\prime}+a u=y$ or $u=\phi, u^{\prime}-b u=-\varphi$, then (1.1) can be written as

$$
\begin{aligned}
& \left\{\begin{array}{l}
x^{\prime}(t)=-a x(t)+y(t), \quad t \neq t_{k}, \\
y^{\prime}(t)=b y(t)-F(t, x(t)), \quad t \neq t_{k}, \\
\Delta x\left(t_{k}\right)=I_{k}\left(x\left(t_{k}\right)\right), \\
\Delta y\left(t_{k}\right)=H_{k}\left(x\left(t_{k}\right)\right), \quad k \in \mathbb{Z},
\end{array}\right. \\
& \left\{\begin{array}{l}
\phi^{\prime}(t)=b \phi(t)-\varphi(t), \quad t \neq t_{k}, \\
\varphi^{\prime}(t)=-a \varphi(t)+F(t, \phi(t)), \quad t \neq t_{k}, \\
\Delta \phi\left(t_{k}\right)=I_{k}\left(\phi\left(t_{k}\right)\right), \\
\Delta \varphi\left(t_{k}\right)=D_{k}\left(\phi\left(t_{k}\right)\right), \quad k \in \mathbb{Z},
\end{array}\right.
\end{aligned}
$$

where $F(t, u)=f(t, u)+a b u, H_{k}(u)=J_{k}(u)+a I_{k}(u)$ and $D_{k}(u)=b I_{k}(u)-J_{k}(u)$.

If $u(t)$ is a $T$-periodic solution of (1.1), then $x, y, \phi, \varphi \in P C^{1}(\mathbb{R})$ are $T$-periodic functions, and $(x, y),(\phi, \varphi)$ satisfy $(2.1),(2.2)$, respectively.

A function pair $(x, y) \in\left\{\left(u_{1}, u_{2}\right) \in P C^{1}(\mathbb{R}, \mathbb{R}) \times P C^{1}(\mathbb{R}, \mathbb{R})\right\}$ and $x, y$ satisfy (2.1) (or (2.2)), we call $z=(x, y)$ a solution of (2.1) (or (2.2)). If $z=(x, y)$ is a solution of (2.1) (or (2.2)) and $x$, $y$ are $T$-periodic, then $x$ is a $T$-periodic solution of (1.1). If $(x, y)$ and $(\phi, \varphi)$ are $T$-periodic solutions of (2.1) and (2.2), respectively, similar to Lemma 2.2 in [12], one can obtain that 
$x, y$ and $\phi, \varphi$ satisfy the integral equation system

$$
\begin{aligned}
& x(t)=\int_{t}^{t+T} G_{a}(t, s) y(s) d s+\sum_{t \leq t_{k}<t+T} G_{a}\left(t, t_{k}\right) I_{k}\left(x\left(t_{k}\right)\right), \\
& y(t)=\int_{t}^{t+T} G_{b}^{*}(t, s) F(s, x(s)) d s-\sum_{t \leq t_{k}<t+T} G_{b}^{*}\left(t, t_{k}\right) H_{k}\left(x\left(t_{k}\right)\right), \\
& \phi(t)=\int_{t}^{t+T} G_{b}^{*}(t, s) \varphi(s) d s-\sum_{t \leq t_{k}<t+T} G_{b}^{*}\left(t, t_{k}\right) I_{k}\left(\phi\left(t_{k}\right)\right), \\
& \varphi(t)=\int_{t}^{t+T} G_{a}(t, s) F(s, \phi(s)) d s+\sum_{t \leq t_{k}<t+T} G_{a}\left(t, t_{k}\right) D_{k}\left(\phi\left(t_{k}\right)\right),
\end{aligned}
$$

where

$$
G_{\lambda}(t, s)=\frac{e^{\lambda(s-t)}}{e^{\lambda T}-1}, \quad G_{\lambda}^{*}(t, s)=\frac{e^{\lambda(t+T-s)}}{e^{\lambda T}-1} .
$$

Lemma 2.1 [14] Let $X$ be a Banach space and $P$ be a cone in $X$. Suppose that $\Omega_{1}$ and $\Omega_{2}$ are open subsets of $X$ such that $0 \in \Omega_{1} \subset \bar{\Omega}_{1} \subset \Omega_{2}$ and suppose that

$$
\Phi: P \cap\left(\bar{\Omega}_{2} \backslash \Omega_{1}\right) \rightarrow P
$$

is a completely continuous operator such that

(i) $\inf \|\Phi u\|>0, u \neq \mu \Phi u$ for $u \in P \cap \partial \Omega_{1}$ and $\mu \geq 1$, and $u \neq \mu \Phi u$ for $u \in P \cap \partial \Omega_{2}$ and $0<\mu \leq 1$, or

(ii) $\inf \|\Phi u\|>0, u \neq \mu \Phi u$ for $u \in P \cap \partial \Omega_{2}$ and $\mu \geq 1$, and $u \neq \mu \Phi u$ for $u \in P \cap \partial \Omega_{1}$ and $0<\mu \leq 1$.

Then $\Phi$ has a fixed point in $P \cap\left(\bar{\Omega}_{2} \backslash \Omega_{1}\right)$.

Set $\delta=e^{-a T}, \sigma=e^{-b T}$ and

$$
\begin{aligned}
& S\left(v_{1}, v_{2}, \ldots, v_{p}\right)=-\sum_{k=1}^{p}\left[J_{k}\left(v_{k}\right)+(a-b) I_{k}\left(v_{k}\right)\right], \quad v_{1}, \ldots, v_{p} \in \mathbb{R}, \gamma=\delta \text { or } \sigma, \\
& \varphi_{\gamma}(s)=\int_{0}^{T} \sup _{u \in[\gamma s, s]} f(t, u) d t+\sup \left\{S\left(v_{1}, v_{2}, \ldots, v_{p}\right): v_{k} \in[\gamma s, s], 1 \leq k \leq p\right\}, \\
& \psi_{\gamma}(s)=\int_{0}^{T} \inf _{u \in[\gamma s, s]} f(t, u) d t+\inf \left\{S\left(u, v_{1}, v_{2}, \ldots, v_{p}\right): v_{k} \in[\gamma s, s], 1 \leq k \leq p\right\} .
\end{aligned}
$$

The following theorems are the main results of this paper.

Theorem 2.1 Assume that $(\mathrm{P})$ holds and there exist two positive constants $r<R$ such that

$$
\begin{aligned}
& F \in C(J \times[\delta r, R],[0,+\infty)), \\
& I_{k} \in C([\delta r, R],[0,+\infty)) \quad(1 \leq k \leq p), \\
& H_{k} \in C([\delta r, R],(-\infty, 0]) \quad(1 \leq k \leq p),
\end{aligned}
$$


where $F(t, u)=f(t, u)+a b u$ and $H_{k}=J_{k}+a I_{k}$. Further suppose that $\varphi_{\delta}(r)<0<\psi_{\delta}(R)$ or $\varphi_{\delta}(R)<0<\psi_{\delta}(r)$, then (1.1) has at least one positive T-periodic solution $x$ with $r \leq\|x\| \leq R$ and $x(t) \geq \delta$ for all $t \in \mathbb{R}$.

Remark 2.1 The condition $\varphi_{\delta}(r)<0<\psi_{\delta}(R)\left(\right.$ or $\left.\varphi_{\delta}(R)<0<\psi_{\delta}(r)\right)$ in Theorem 2.1 can be replaced by the condition easily verified

$$
\Upsilon(r)<0<\gamma(R) \quad(\text { or } \Upsilon(R)<0<\gamma(r)),
$$

where

$$
\begin{aligned}
& \Upsilon(s)=T \sup _{(t, u) \in[0, T] \times[\delta s, s]} f(t, u)+\sup \left\{S\left(v_{1}, v_{2}, \ldots, v_{p}\right): v_{k} \in[\delta s, s], 1 \leq k \leq p\right\}, \\
& \gamma(s)=T \inf _{(t, u) \in[0, T] \times[\delta s, s]} f(t, u)+\inf \left\{S\left(v_{1}, v_{2}, \ldots, v_{p}\right): v_{k} \in[\delta s, s], 1 \leq k \leq p\right\} .
\end{aligned}
$$

Proof Here we only prove the case $\varphi_{\delta}(r)<0<\psi_{\delta}(R)$. Let

$$
\begin{aligned}
& \tilde{f}(t, u)=\left\{\begin{array}{l}
f(t, \delta r) \text { if } u<\delta r, \\
f(t, u) \text { if } \delta r \leq u \leq R, \\
f(t, R) \text { if } u>R,
\end{array}\right. \\
& \tilde{I}_{k}(u)=\left\{\begin{array}{ll}
I_{k}(\delta r) \text { if } u<\delta r, \\
I_{k}(u) \text { if } \delta r \leq u \leq R, \\
I_{k}(R) \text { if } u>R,
\end{array} \quad \tilde{J}_{k}(u)= \begin{cases}J_{k}(\delta r) & \text { if } u<\delta r, \\
J_{k}(u) & \text { if } \delta r \leq u \leq R, \\
J_{k}(R) & \text { if } u>R,\end{cases} \right. \\
& \tilde{F}(t, u)=\tilde{f}(t, u)+a b u, \quad \tilde{H}_{k}(u)=\tilde{J}_{k}(u)+a \tilde{I}_{k}(u), \\
& \tilde{S}\left(v_{1}, \ldots, v_{p}\right)=\sum_{k=1}^{p} \tilde{S}_{k}\left(v_{k}\right), \quad \tilde{S}_{k}(v)=-\tilde{J}_{k}(v)-(a-b) \tilde{I}_{k}(v) .
\end{aligned}
$$

At first, we show that

$$
\int_{0}^{T} \sup _{\delta s \leq u \leq s} \tilde{f}(t, u) d t+\sup \left\{\tilde{S}\left(v_{1}, \ldots, v_{p}\right): \delta s \leq v_{k} \leq s\right\}<0, \quad \forall s \in(0, r]
$$

We claim that

$$
\sup _{\delta s \leq u \leq s} \tilde{f}(t, u) \leq \sup _{\delta r \leq u \leq r} \tilde{f}(t, u)=\sup _{\delta r \leq u \leq r} f(t, u), \quad s \in(0, r]
$$

In fact, if $s \leq \delta r, \sup _{\delta s \leq u \leq s} \tilde{f}(t, u)=\sup _{\delta s \leq u \leq s} f(t, \delta r)=f(t, \delta r) \leq \sup _{\delta r \leq u \leq r} f(t, u)$. If $\delta r \leq$ $s \leq r$, there exists $u_{t} \in[\delta s, s]$ such that $\tilde{f}\left(t, u_{t}\right)=\sup _{\delta s \leq u \leq s} \tilde{f}(t, u)$. We consider two subcases.

Subcase 1: $\delta r \leq u_{t} \leq s . \tilde{f}\left(t, u_{t}\right) \leq \sup _{\delta r \leq u \leq r} \tilde{f}(t, u)$.

Subcase 2: $\delta s \leq u_{t} \leq \delta r . \tilde{f}\left(t, u_{t}\right)=f(t, \delta r) \leq \sup _{\delta r \leq u \leq r} f(t, u)$.

Hence, (2.4) holds and

$$
\int_{0}^{T} \sup _{\delta s \leq u \leq s} \tilde{f}(t, u) d t \leq \int_{0}^{T} \sup _{\delta r \leq u \leq r} f(t, u) d t, \quad s \in(0, r] .
$$


Similar to (2.4), we obtain that

$$
\begin{aligned}
\sup _{\delta s \leq v \leq s} \tilde{S}_{k}(v) \leq \sup _{\delta r \leq v \leq r} \tilde{S}_{k}(v), \quad \forall s \in(0, r] & \\
\sup _{\delta s \leq v_{k} \leq s} \tilde{S}\left(v_{1}, \ldots, v_{p}\right) & =\sup _{\delta s \leq v_{k} \leq s} \sum_{k=1}^{p} \tilde{S}_{k}\left(v_{k}\right)=\sum_{k=1}^{p} \sup _{\delta s \leq v \leq s} \tilde{S}_{k}(v) \\
& \leq \sum_{k=1}^{p} \sup _{\delta r \leq v \leq r} \tilde{S}_{k}(v)=\sup _{\delta r \leq v_{k} \leq r} \sum_{k=1}^{p} \tilde{S}_{k}\left(v_{k}\right) \\
& =\sup _{\delta r \leq v_{k} \leq r} \tilde{S}\left(v_{1}, \ldots, v_{p}\right)=\sup _{\delta r \leq v_{k} \leq r} S\left(v_{1}, \ldots, v_{p}\right), \quad \forall s \in(0, r] .
\end{aligned}
$$

Thus

$$
\int_{0}^{T} \sup _{\delta s \leq u \leq s} \tilde{f}(t, u) d t+\sup \left\{\tilde{S}\left(v_{1}, \ldots, v_{p}\right): \delta s \leq v_{k} \leq s\right\} \leq \varphi_{\delta}(r)<0, \quad \forall s \in(0, r]
$$

Next, we define operator and cone. Let $E=\{z=(x, y) \in P C(\mathbb{R}) \times P C(\mathbb{R}), x(t+T)=$ $x(t), y(t+T)=y(t)\}$, then $E$ is a Banach space with the norm $\|z\|_{E}=\|x\|+\|y\|$, where $\|x\|=\max _{t \in[0, T]}|x(t)|$ and $\|y\|=\max _{t \in[0, T]}|y(t)|$.

Define the mapping $A: E \rightarrow E$ and the cone $P$ in $E$ by

$$
A(x, y)=(X, Y)
$$

where

$$
\begin{aligned}
& X(t)=\int_{t}^{t+T} G_{a}(t, s) y(s) d s+\sum_{t \leq t_{k}<t+T} G_{a}\left(t, t_{k}\right) \tilde{I}_{k}\left(x\left(t_{k}\right)\right), \\
& Y(t)=\int_{t}^{t+T} G_{b}^{*}(t, s) \tilde{F}(s, x(s)) d s-\sum_{t \leq t_{k}<t+T} G_{b}^{*}\left(t, t_{k}\right) \tilde{H}_{k}\left(x\left(t_{k}\right)\right), \\
& P=\{z=(x, y) \in E: x(t) \geq \delta\|x\|, y(t) \geq \sigma\|y\|, t \in \mathbb{R}\} .
\end{aligned}
$$

Put

$$
\begin{aligned}
& \Omega_{1}=\left\{z=(x, y) \in E:\|x\|<r,\|y\|<\varepsilon+a \sigma^{-1} r\right\}, \\
& \Omega_{2}=\left\{z \in E:\|x\|<R,\|y\|<\varepsilon+a \sigma^{-1} R\right\},
\end{aligned}
$$

where $0<\varepsilon<a \sigma^{-1}(R-r)$. At first, we show that $A: P \cap\left(\bar{\Omega}_{2} \backslash \Omega_{1}\right) \rightarrow P$. For any $(x, y) \in P$, it is easy to verify that $A(x, y)(t+T)=A(x, y)(t)$, that is, $X(t+T)=X(t)$ and $Y(t+T)=Y(t)$. We need to show that $A(x, y)(t)=(X(t), Y(t)) \in P$ for $(x, y) \in P$, that is, $X(t) \geq \delta\|X\|, Y(t) \geq$ $\sigma\|Y\|$ for any $t \in[0, T]$. Noting that

$$
\begin{aligned}
X(t) & \leq \frac{e^{a T}}{e^{a T}-1} \int_{t}^{t+T} y(s) d s+\frac{e^{a T}}{e^{a T}-1} \sum_{t \leq t_{k}<t+T} \tilde{I}_{k}\left(x\left(t_{k}\right)\right) \\
& =\frac{e^{a T}}{e^{a T}-1}\left(\int_{0}^{T} y(s) d s+\sum_{k=1}^{p} \tilde{I}_{k}\left(x\left(t_{k}\right)\right)\right),
\end{aligned}
$$




$$
\begin{aligned}
X(t) & \geq \frac{1}{e^{a T}-1}\left(\int_{0}^{T} y(s) d s+\sum_{k=1}^{p} \tilde{I}_{k}\left(x\left(t_{k}\right)\right)\right), \\
Y(t) & \leq \frac{e^{b T}}{e^{b T}-1} \int_{t}^{t+T} \tilde{F}(s, x(s)) d s-\frac{e^{b T}}{e^{b T}-1} \sum_{t \leq t_{k}<t+T} \tilde{H}_{k}\left(x\left(t_{k}\right)\right) \\
& =\frac{e^{b T}}{e^{b T}-1}\left(\int_{0}^{T} \tilde{F}(s, x(s)) d s-\sum_{k=1}^{p} \tilde{H}_{k}\left(x\left(t_{k}\right)\right)\right), \\
Y(t) & \geq \frac{1}{e^{b T}-1}\left(\int_{0}^{T} \tilde{F}(s, x(s)) d s-\sum_{k=1}^{p} \tilde{H}_{k}\left(x\left(t_{k}\right)\right)\right),
\end{aligned}
$$

we obtain that $X(t) \geq \delta\|X\|$ and $Y(t) \geq \sigma\|Y\|$, and hence $A(P) \subset P$. In addition, one can easily check that $A: P \cap\left(\bar{\Omega}_{2} \backslash \Omega_{1}\right) \rightarrow P$ is completely continuous.

Finally, we show that the condition (ii) of Lemma 2.1 is satisfied. We firstly show

$$
z \neq \mu A z, \quad \forall z \in P \cap \partial \Omega_{1}, 0<\mu \leq 1 .
$$

If not, there exist $z_{0}=\left(x_{0}, y_{0}\right) \in P \cap \partial \Omega_{1}$ and $0<\mu_{0} \leq 1$ such that $z_{0}=\mu_{0} A z_{0}$. Then

$$
\left\{\begin{array}{l}
x_{0}^{\prime}(t)=-a x_{0}(t)+\mu_{0} y_{0}(t), \quad t \neq t_{k}, \\
y_{0}^{\prime}(t)=b y_{0}(t)-\mu_{0} \tilde{F}\left(t, x_{0}(t)\right), \quad t \neq t_{k}, \\
\Delta x_{0}\left(t_{k}\right)=\mu_{0} \tilde{I}_{k}\left(x_{0}\left(t_{k}\right)\right), \\
\Delta y_{0}\left(t_{k}\right)=\mu_{0} \tilde{H}_{k}\left(x_{0}\left(t_{k}\right)\right), \quad k \in \mathbb{Z} .
\end{array}\right.
$$

By integration, we obtain that

$$
\begin{aligned}
& a \int_{0}^{T} x_{0}(s) d s=\mu_{0} \int_{0}^{T} y_{0}(s) d s+\mu_{0} \sum_{k=1}^{p} \tilde{I}_{k}\left(x_{0}\left(t_{k}\right)\right) \\
& b \int_{0}^{T} y_{0}(s) d s+\mu_{0} \sum_{k=1}^{p} \tilde{H}_{k}\left(x_{0}\left(t_{k}\right)\right)=\mu_{0} \int_{0}^{T} \tilde{F}\left(s, x_{0}(s)\right) d s .
\end{aligned}
$$

From (2.6) and (2.7), we have

$$
\begin{aligned}
& \int_{0}^{T} \tilde{f}\left(s, x_{0}(s)\right) d s-\sum_{k=1}^{p}\left[\tilde{J}_{k}\left(x_{0}\left(t_{k}\right)\right)+(a-b) \tilde{I}_{k}\left(x_{0}\left(t_{k}\right)\right)\right] \\
& \quad=a b\left(\mu_{0}^{-1}-1\right) \int_{0}^{T} x_{0}(s) d s+b\left(\mu_{0}^{-1}-1\right) \int_{0}^{T} y_{0}(s) d s \geq 0 .
\end{aligned}
$$

Since $z_{0}=\left(x_{0}, y_{0}\right) \in P \cap \partial \Omega_{1},\left\|x_{0}\right\|=r$ or $\left\|y_{0}\right\|=\varepsilon+a \sigma^{-1} r$. If $\left\|x_{0}\right\|=0$, one easily gets that $y_{0}=0, t \neq t_{k}$. On the other hand, the fact that $\left\|y_{0}\right\|=\varepsilon+a \sigma^{-1} r$ and $y_{0}$ is left continuous implies that $y_{0} \not \equiv 0$ for $t \neq t_{k}$, a contradiction. Thus $0<\left\|x_{0}\right\| \leq r$. Noting that $\int_{0}^{T} \tilde{f}\left(s, x_{0}(s)\right) d s \leq \int_{0}^{T} \sup _{\delta\left\|x_{0}\right\| \leq u \leq\left\|x_{0}\right\|} \tilde{f}(s, u) d s$, we obtain that

$$
\int_{0}^{T} \sup _{\delta\left\|x_{0}\right\| \leq u \leq\left\|x_{0}\right\|} \tilde{f}(s, u) d s-\sum_{k=1}^{p}\left[\tilde{J}_{k}\left(x_{0}\left(t_{k}\right)\right)+(a-b) \tilde{I}_{k}\left(x_{0}\left(t_{k}\right)\right)\right] \geq 0,
$$


which is in contradiction with (2.3). Hence,

$$
z \neq \mu A z, \quad z \in P \cap \partial \Omega_{1}, 0<\mu \leq 1 .
$$

We show that for $z \in P \cap \partial \Omega_{2}$ and $\mu \geq 1$,

$$
\inf \|A z\|_{E}>0 \quad \text { and } \quad z \neq \mu A z \text {. }
$$

Assume that $\inf _{z \in P \cap \partial \Omega_{2}}\|A z\|_{E}=0$. There exists the sequence $z_{n}=\left(x_{n}, y_{n}\right) \in P \cap \partial \Omega_{2}$ such that $\left\|A\left(x_{n}, y_{n}\right)=\left(X_{n}, Y_{n}\right)\right\|_{E} \rightarrow 0$ as $n \rightarrow \infty$. Hence, $x_{n} \geq \delta\left\|x_{n}\right\|, y_{n} \geq \sigma\left\|y_{n}\right\|,\left\|x_{n}\right\|=R$ or $\left\|y_{n}\right\|=\varepsilon+a \sigma^{-1} R$ and

$$
\begin{aligned}
& \frac{1}{e^{a T}-1}\left(\int_{0}^{T} y_{n}(s) d s+\sum_{k=1}^{p} \tilde{I}_{k}\left(x_{n}\left(t_{k}\right)\right)\right) \leq\left\|X_{n}(t)\right\| \rightarrow 0, \\
& \frac{1}{e^{b T}-1}\left(\int_{0}^{T} \tilde{F}\left(s, x_{n}(s)\right) d s-\sum_{k=1}^{p} \tilde{H}_{k}\left(x_{n}\left(t_{k}\right)\right)\right) \leq\left\|Y_{n}(t)\right\| \rightarrow 0
\end{aligned}
$$

as $n \rightarrow \infty$, which imply that

$$
\begin{aligned}
& 0 \leq \sigma T\left\|y_{n}\right\| \leq \int_{0}^{T} y_{n}(t) d t \rightarrow 0, \quad \tilde{I}_{k}\left(x_{n}\left(t_{k}\right)\right) \rightarrow 0 \quad(1 \leq k \leq p), \\
& \int_{0}^{T} \tilde{F}\left(s, x_{n}(s)\right) d s-\sum_{k=1}^{p} \tilde{H}_{k}\left(x_{n}\left(t_{k}\right)\right) \rightarrow 0
\end{aligned}
$$

as $n \rightarrow \infty$. Hence $\left\|x_{n}\right\|=R$ and $x_{n}(t) \geq \delta\left\|x_{n}\right\|=\delta R$. We have

$$
\begin{aligned}
0 & <\psi_{\delta}(R) \leq \int_{0}^{T} \tilde{f}\left(t, x_{n}(t)\right) d t-\sum_{k=1}^{p}\left[\tilde{I}_{k}\left(x_{n}\left(t_{k}\right)\right)+(a-b) \tilde{I}_{k}\left(x_{n}\left(t_{k}\right)\right)\right] \\
& \leq \int_{0}^{T} \tilde{F}\left(s, x_{n}(s)\right) d s-\sum_{k=1}^{p} \tilde{H}_{k}\left(x_{n}\left(t_{k}\right)\right)+b \sum_{k=1}^{p} \tilde{I}_{k}\left(x_{n}\left(t_{k}\right)\right) \rightarrow 0, \quad n \rightarrow \infty,
\end{aligned}
$$

which is a contradiction.

Suppose that there exist $z_{1}=\left(x_{1}, y_{1}\right) \in P \cap \partial \Omega_{2}$ and $\mu_{1} \geq 1$ such that $z_{1}=\mu_{1} A z_{1}$. Then

$$
\left\{\begin{array}{l}
x_{1}^{\prime}(t)=-a x_{1}(t)+\mu_{1} y_{1}(t), \quad t \neq t_{k}, \\
y_{1}^{\prime}(t)=a y_{1}(t)-\mu_{1} \tilde{F}\left(t, x_{1}(t)\right), \quad t \neq t_{k}, \\
\Delta x_{1}\left(t_{k}\right)=\mu_{1} \tilde{I}_{k}\left(x_{1}\left(t_{k}\right)\right), \\
\Delta y_{1}\left(t_{k}\right)=\mu_{1} \tilde{H}_{k}\left(x_{1}\left(t_{k}\right)\right), \quad k \in \mathbb{Z} .
\end{array}\right.
$$

Similar to (2.6) and (2.7), we have

$$
\begin{aligned}
& a \int_{0}^{T} x_{1}(s) d s=\mu_{1} \int_{0}^{T} y_{1}(s) d s+\mu_{1} \sum_{k=1}^{p} \tilde{I}_{k}\left(x_{1}\left(t_{k}\right)\right), \\
& b \int_{0}^{T} y_{1}(s) d s+\mu_{1} \sum_{k=1}^{p} \tilde{H}_{k}\left(x_{1}\left(t_{k}\right)\right)=\mu_{1} \int_{0}^{T} \tilde{F}\left(s, x_{1}(s)\right) d s,
\end{aligned}
$$




$$
\begin{aligned}
& a b\left(1-\mu_{1}\right) \int_{0}^{T} x_{1}(s) d s+b\left(1-\mu_{1}\right) \int_{0}^{T} y_{1}(s) d s \\
& \quad=\mu_{1}\left(\int_{0}^{T} \tilde{f}\left(s, x_{1}(s)\right) d s-\sum_{k=1}^{p}\left[\tilde{J}_{k}\left(x_{1}\left(t_{k}\right)\right)+(a-b) \tilde{I}_{k}\left(x_{1}\left(t_{k}\right)\right)\right]\right) .
\end{aligned}
$$

We consider two cases.

Case 1 If $\left\|x_{1}\right\|=R$, then $x_{1} \geq \delta\left\|x_{1}\right\| \geq \delta R$. Noting that $x_{1} \geq 0, y_{1} \geq 0$ for all $t \in J$ and $\mu_{1} \geq 1$, we obtain that

$$
\int_{0}^{T} \tilde{f}\left(s, x_{1}(s)\right) d s-\sum_{k=1}^{p}\left[\tilde{J}_{k}\left(x_{1}\left(t_{k}\right)\right)+(a-b) \tilde{I}_{k}\left(x_{1}\left(t_{k}\right)\right)\right] \leq 0,
$$

which is in contradiction with the fact $\psi_{\delta}(R)>0$.

Case 2 If $\left\|x_{1}\right\|<R$, then $\left\|y_{1}\right\|=\varepsilon+a \sigma^{-1} R$. From (2.9) and $\tilde{I}_{k} \geq 0$, we have

$$
a T R \geq a T\left\|x_{1}\right\| \geq a \int_{0}^{T} x_{1}(s) d s \geq \mu_{1} \int_{0}^{T} y_{1}(s) d s \geq T \sigma\left\|y_{1}\right\|
$$

which implies that $\left\|y_{1}\right\| \leq a \sigma^{-1} R$, a contradiction.

The condition (ii) of Lemma 2.1 is fulfilled and it follows that $A$ has at least one fixed point $z=(x, y) \in P \cap\left(\bar{\Omega}_{2} \backslash \Omega_{1}\right)$. Clearly, $x$, $y$ satisfy

$$
\left\{\begin{array}{l}
x^{\prime}(t)=-a x(t)+y(t), \quad t \neq t_{k}, \\
y^{\prime}(t)=a y(t)-\tilde{F}(t, x(t)), \quad t \neq t_{k}, \\
\Delta x\left(t_{k}\right)=\tilde{I}_{k}\left(x\left(t_{k}\right)\right), \\
\Delta y\left(t_{k}\right)=\tilde{H}_{k}\left(x\left(t_{k}\right)\right), \quad k \in \mathbb{Z} .
\end{array}\right.
$$

Suppose that $\|x\|<r$. By integrating the first equation of (2.12), we obtain that

$$
a \operatorname{Tr} \geq a T\|x\| \geq a \int_{0}^{T} x(s) d s \geq \int_{0}^{T} y(s) d s \geq T \sigma\|y\|,
$$

which implies that $\|y\| \leq a \sigma^{-1} r$, a contradiction to $(x, y) \in P \cap\left(\bar{\Omega}_{2} \backslash \Omega_{1}\right)$. Hence, $r \leq\|x\| \leq R$, $\tilde{f}(t, x(t))=f(t, x(t)), \tilde{I}_{k}\left(x\left(t_{k}\right)\right)=I_{k}\left(x\left(t_{k}\right)\right), \tilde{J}_{k}\left(x\left(t_{k}\right)\right)=J_{k}\left(x\left(t_{k}\right)\right)$, and

$$
\left\{\begin{array}{l}
x^{\prime}(t)=-a x(t)+y(t), \quad t \neq t_{k}, \\
y^{\prime}(t)=a y(t)-F(t, x(t)), \quad t \neq t_{k}, \\
\Delta x\left(t_{k}\right)=I_{k}\left(x\left(t_{k}\right)\right), \\
\Delta y\left(t_{k}\right)=H_{k}\left(x\left(t_{k}\right)\right), \quad k \in \mathbb{Z} .
\end{array}\right.
$$

It is easy to check that $x$ is one positive $T$-periodic solution of (1.1). The proof is complete.

We introduce the following assumptions:

$$
\begin{aligned}
& \lim _{x \rightarrow 0^{+}} \frac{J_{k}(x)+(a-b) I_{k}(x)}{x^{m}}=\alpha_{k}(m), \quad \lim _{x \rightarrow+\infty} \frac{J_{k}(x)+(a-b) I_{k}(x)}{x^{m}}=\beta_{k}(m), \\
& \liminf _{x \rightarrow 0^{+}} \min _{t \in[0, T]} \frac{f(t, x)}{x^{m}}=h(m), \quad \limsup _{x \rightarrow 0^{+}} \max _{t \in[0, T]} \frac{f(t, x)}{x^{m}}=\bar{h}(m),
\end{aligned}
$$


$\liminf _{x \rightarrow+\infty} \min _{t \in[0, T]} \frac{f(t, x)}{x^{m}}=g(m), \quad \limsup _{x \rightarrow+\infty} \max _{t \in[0, T]} \frac{f(t, x)}{x^{m}}=\bar{g}(m)$.

$\left(C_{1}\right)$ There exist $n, m_{k} \in[0, \infty)(1 \leq k \leq p)$ such that $-\infty<\alpha_{k}\left(m_{k}\right) \leq 0,-\infty<\bar{h}(n)<0$, $n<\min \left\{m_{1}, \ldots, m_{p}\right\}$.

$\left(C_{2}\right)$ There exist $0 \leq k_{0} \leq p, l_{1}, l_{2}: 0 \leq l_{1}<l_{2}$ such that $-\infty<\beta_{k_{0}}\left(l_{2}\right)<0, g\left(l_{1}\right)>-\infty$.

$\left(C_{3}\right)$ There exists a constant $m \geq 0$ such that $g(m)>0$.

$\left(C_{4}\right)$ There exist $n, m_{k} \in[0, \infty)(1 \leq k \leq p)$ such that $-\infty<\beta_{k}\left(m_{k}\right) \leq 0,-\infty<\bar{g}(n)<0$, $n>\max \left\{m_{1}, \ldots, m_{p}\right\}$.

$\left(C_{5}\right)$ There exist $0 \leq k_{0} \leq p, l_{1}, l_{2}: 0 \leq l_{1}<l_{2}$ such that $-\infty<\alpha_{k_{0}}\left(l_{1}\right)<0, h\left(l_{2}\right)>-\infty$.

$\left(C_{6}\right)$ There exists a constant $m \geq 0$ such that $h(m)>0$.

Corollary 2.1 Assume that $F \in C(J \times[0,+\infty),[0,+\infty)), I_{k} \in C([0,+\infty),[0,+\infty))(1 \leq k \leq$ $p), H_{k} \in C([0,+\infty),(-\infty, 0])(1 \leq k \leq p)$. Then (1.1) has at least one positive T-periodic solution if one of the following conditions is satisfied:

(1) $\left(\mathrm{C}_{1}\right)$ and $\left(\mathrm{C}_{2}\right)$;

(2) $\left(\mathrm{C}_{1}\right)$ and $\left(\mathrm{C}_{3}\right)$;

(3) $\left(\mathrm{C}_{4}\right)$ and $\left(\mathrm{C}_{5}\right)$;

(4) $\left(\mathrm{C}_{4}\right)$ and $\left(\mathrm{C}_{6}\right)$.

Proof We only consider case (1). By $\left(\mathrm{C}_{1}\right)$ and $\left(\mathrm{C}_{2}\right)$, there exist $R_{1}>1>r_{1}>0$ such that

$$
\begin{aligned}
& J_{k}(x)+(a-b) I_{k}(x) \geq\left(\alpha_{k}\left(m_{k}\right)+\bar{h}(n)\right) x^{m_{k}}, \quad \forall 0<x \leq r_{1}, \\
& f(t, x) \leq \frac{1}{2} \bar{h}(n) x^{n}, \quad \forall t \in R, 0<x \leq r_{1}, \\
& J_{k_{0}}(x)+(a-b) I_{k_{0}}(x) \leq \frac{1}{2} \beta_{k_{0}}\left(l_{2}\right) x^{l_{2}}, \quad x \geq R_{1}, \\
& f(t, x) \geq \frac{1}{2} g\left(l_{1}\right) x^{l_{1}}, \quad \forall t \in R, x \geq R_{1} .
\end{aligned}
$$

Choosing

$$
\begin{aligned}
& r=\frac{1}{2} \min \left\{r_{1},\left(\frac{-T \bar{h}(n) \delta^{n}}{2 \sum_{k=1}^{p}\left|\alpha_{k}\left(m_{k}\right)+\bar{h}(n)\right|}\right)^{\frac{1}{\min \left\{m_{1}, m_{2}, \ldots m_{p}\right\}-n}}\right\}, \\
& R=2 \max \left\{R_{1} / \delta,\left(\frac{\left|g\left(l_{1}\right)\right| T}{-\beta_{k_{0}}\left(l_{2}\right) \delta^{l_{2}}}\right)^{\frac{1}{l_{2}-l_{1}}}\right\},
\end{aligned}
$$

we have

$$
\begin{aligned}
& \int_{0}^{T} \sup _{\delta r \leq u \leq r} f(t, u) d t-\sum_{k=1}^{p}\left[J_{k}\left(v_{k}\right)+(a-b) I_{k}\left(v_{k}\right)\right] \\
& \quad \leq \frac{1}{2} \bar{h}(n) T(\delta r)^{n}+\sum_{k=1}^{p}\left|\alpha_{k}\left(m_{k}\right)+\bar{h}(n)\right| r^{m_{k}} \\
& \quad \leq \frac{1}{2} \bar{h}(n) T(\delta r)^{n}+r^{m} \sum_{k=1}^{p}\left|\alpha_{k}\left(m_{k}\right)+\bar{h}(n)\right|<0,
\end{aligned}
$$


where $m=\min \left\{m_{1}, m_{2}, \ldots, m_{p}\right\}$ and $v_{k} \in[\delta r, r]$, and

$$
\int_{0}^{T} \inf _{\delta R \leq u \leq R} f(t, u) d t-\sum_{k=1}^{p}\left[J_{k}\left(v_{k}\right)+(a-b) I_{k}\left(v_{k}\right)\right] \geq-\frac{1}{2}\left|g\left(l_{1}\right)\right| T R^{l_{1}}+\frac{1}{2}\left|\beta_{k_{0}}\left(l_{2}\right)\right|(\delta R)^{l_{2}}>0
$$

for $v_{k} \in[\delta R, R]$. By Theorem 2.1, (1.1) has at least one $T$-periodic solution $x$ with $r \leq\|x\| \leq R$.

Remark 2.2 From Corollary 2.1, we easily obtain Theorem 3.1 and Theorem 3.2 in [12]. Moreover, the conditions of cases (1) and (3) are weaker than those of Theorem 3.1 and Theorem 3.2 in [12].

Define the mapping $B: E \rightarrow E$ by

$$
B(x, y)=(\hat{X}, \hat{Y})
$$

where

$$
\begin{aligned}
& \hat{X}(t)=\int_{t}^{t+T} G_{b}^{*}(t, s) y(s) d s-\sum_{t \leq t_{k}<t+T} G_{b}^{*}\left(t, t_{k}\right) \tilde{I}_{k}\left(x\left(t_{k}\right)\right), \\
& \hat{Y}(t)=\int_{t}^{t+T} G_{a}(t, s) \tilde{F}(s, x(s)) d s+\sum_{t \leq t_{k}<t+T} G_{a}\left(t, t_{k}\right)\left(b \tilde{I}_{k}\left(x\left(t_{k}\right)\right)-\tilde{J}_{k}\left(x\left(t_{k}\right)\right)\right) .
\end{aligned}
$$

Similar to Theorem 2.1, we have the following result.

Theorem 2.2 Assume that $(\mathrm{P})$ holds and there exist two positive constants $r<R$ such that

$$
\begin{aligned}
& F \in C(J \times[\sigma r, R],[0,+\infty)), \\
& I_{k} \in C([\sigma r, R],(-\infty, 0]) \quad(1 \leq k \leq p), \\
& D_{k} \in C([\sigma r, R],[0,+\infty)) \quad(1 \leq k \leq p),
\end{aligned}
$$

where $F(t, u)=f(t, u)+a b u$ and $D_{k}=b I_{k}-J_{k}$. Further suppose that $\varphi_{\sigma}(r)<0<\psi_{\sigma}(R)$ or $\varphi_{\sigma}(R)<0<\psi_{\sigma}(r)$ is satisfied, then (1.1) has at least one positive T-periodic solution $x$ with $r \leq\|x\| \leq R$ and $x(t) \geq$ or for all $t \in \mathbb{R}$.

\section{Application}

In this section, some examples are provided to highlight our results obtained in previous section and the results in $[12,13]$ cannot been applied.

Example 3.1 Consider the differential equation

$$
\left\{\begin{array}{l}
u^{\prime \prime}(t)+C u^{\prime}(t)+f(u(t))=0, \quad t \neq t_{k}, \\
\Delta u^{\prime}\left(t_{k}\right)=-J_{k}\left(u\left(t_{k}\right)\right), \quad k \in \mathbb{Z},
\end{array}\right.
$$

where $C \in \mathbb{R}$, and there exists $p \in \mathbb{N}$ such that $J_{k+p}(u)=J_{k}(u), t_{k+p}=t_{k}+T$. Moreover, the following condition holds: 
(PP) there exist constants $\alpha>0,0<\xi<1,0<r<R$ such that

$$
\begin{aligned}
& f \in C([\xi r, R], \mathbb{R}), \quad J_{k} \in C([\xi r, R],[0,+\infty)) \quad(1 \leq k \leq p), \\
& f(u)+\alpha u \geq 0 \quad \text { for } u \in[\xi r, R] \\
& \left(f(r) T+\sum_{k=1}^{p} J_{k}(r)\right)\left(f(R) T+\sum_{k=1}^{p} J_{k}(R)\right)<0 .
\end{aligned}
$$

Proposition 3.1 Problem (3.1) has at least one positive T-periodic solution $x: r \leq\|x\| \leq R$ for $|C|$ sufficiently large.

Proof Without loss of generality, we suppose that

$$
T f(r)+\sum_{k=1}^{p} J_{k}(r)<0, \quad T f(R)+\sum_{k=1}^{p} J_{k}(R)>0 .
$$

Since $f(u), J_{k}(u)$ are continuous in $u \in[\xi r, R]$, there exists $\xi<\tau<1$ such that

$$
\begin{aligned}
& T \sup _{\tau r \leq u \leq r} f(u)+\sum_{k=1}^{p} J_{k}\left(v_{k}\right)<0, \quad \forall \tau r \leq v_{1}, \ldots, v_{p} \leq r, \\
& T \inf _{\tau R \leq u \leq R} f(u)+\sum_{k=1}^{p} J_{k}\left(v_{k}\right)>0, \quad \forall \tau R \leq v_{1}, \ldots, v_{p} \leq R .
\end{aligned}
$$

Choose $b=\alpha C^{-1}, a=b+C$ if $C>0, a=\alpha|C|^{-1}, b=a-C$ if $C<0$, then $a>0, b>0$ and

$$
f(u)+a b u \geq f(u)+\alpha u \geq 0 \quad \text { for } u \in[\tau r, R] .
$$

Note that

$$
\begin{aligned}
& \tau<e^{-b T}<1 \quad \text { for } C>\frac{\alpha T}{-\ln \tau}, \quad \tau<e^{-a T}<1 \quad \text { for } C<\frac{\alpha T}{\ln \tau}, \\
& \int_{0}^{T} \sup _{\nu r \leq u \leq r} f(u) d t+\sum_{k=1}^{p} J_{k}\left(v_{k}\right)=T \sup _{\nu r \leq u \leq r} f(u)+\sum_{k=1}^{p} J_{k}\left(v_{k}\right)<0, \quad \forall v r \leq v_{1}, \ldots, v_{p} \leq r, \\
& \int_{0}^{T} \inf _{\nu R \leq u \leq R} f(t, u) d t+\sum_{k=1}^{p} J_{k}\left(v_{k}\right)=T \inf _{\nu r \leq u \leq r} f(u)+\sum_{k=1}^{p} J_{k}\left(v_{k}\right)>0, \quad \forall v R \leq v_{1}, \ldots, v_{p} \leq R,
\end{aligned}
$$

where $v=e^{-a T}$ if $C<0, v=e^{-b T}$ if $C>0$. By Theorem $2.1(C<0)$ or Theorem $2.2(C>0)$, we obtain that (3.1) has at least one positive $T$-periodic solution $x: r \leq\|x\| \leq R$.

Consider the differential equation

$$
\left\{\begin{array}{l}
u^{\prime \prime}(t)+C u^{\prime}(t)+u(t) \sin u(t)=0, \quad t \neq t_{k} \\
\Delta u^{\prime}\left(t_{k}\right)=-\sqrt[3]{u\left(t_{k}\right)}, \quad k \in \mathbb{Z},
\end{array}\right.
$$

where $C \in \mathbb{R}, t_{k}<t_{k+1}$ and there exists $p \in \mathbb{N}$ such that $t_{k+p}=t_{k}+T$. 
By Proposition 3.1, one can obtain that (3.5) has at least one positive $T$-periodic solution $x: 2 m \pi-0.5 \pi \leq\|x\| \leq 2 m \pi+0.5 \pi$ with sufficiently large $m \in \mathbb{N}$ if $|C|$ is sufficiently large. Thus

$$
\lim _{C \rightarrow+\infty} \lambda^{\sharp}=+\infty,
$$

where $\lambda \sharp$ is the number of positive periodic solutions of (3.5).

Now, consider the special case of (3.5)

$$
\left\{\begin{array}{l}
u^{\prime \prime}(t)+900 u^{\prime}(t)+u \sin u=0, \quad t \neq k \\
\Delta u^{\prime}(k)=-\sqrt[3]{u(k)}, \quad k \in \mathbb{Z}
\end{array}\right.
$$

Let $b=\left(-900+\sqrt{900^{2}+4}\right) / 2, a=900+b, T=1, \sigma=e^{-b}, f(u)=u \sin u, r_{i}^{1}=(2 i-0.5) \pi$, $r_{i}^{2}=(2 i+0.5) \pi, r_{i}^{3}=(2 i+1.5) \pi, i=1,2, \ldots, q=\left[\frac{1}{8 b}-\frac{5}{8}\right]$, where $[d]$ denotes the integer part of $d$. It is easy to check that

$$
f(u)+a b u \geq 0, \quad \forall u \geq 0, \quad(1-\sigma) r_{i}^{j} \leq \pi / 4, \quad j=1,2,3,1 \leq i \leq q,
$$

$f$ is nonincreasing in $\left[\sigma r_{i}^{j}, r_{i}^{j}\right], j=1,3$, and nondecreasing in $\left[\sigma r_{i}^{2}, r_{i}^{2}\right]$.

Hence,

$$
\begin{aligned}
& T \sup u \sin u+\sqrt[3]{v} \leq \sigma r_{i}^{j} \sin \left(\delta r_{i}^{j}\right)+\sqrt[3]{r_{i}^{j}} \leq \sigma r_{i}^{j} \sin \left(r_{i}^{j}-\frac{\pi}{4}\right)+\sqrt[3]{r_{i}^{j}} \\
& \leq-\frac{\sigma \sqrt{2}}{2} r_{i}^{j}+\sqrt[3]{r_{i}^{j}}<0, \quad \sigma r_{i}^{j} \leq u, v \leq r_{i}^{j}, j=1,3, \\
& T \inf u \sin u+\sqrt[3]{v} \geq \sigma r_{i}^{j} \sin \left(\sigma r_{i}^{j}\right) \geq \sigma r_{i}^{j} \sin \left(r_{i}^{j}-\frac{\pi}{4}\right) \geq \frac{\sigma \sqrt{2}}{2} r_{i}^{j}>0, \quad \sigma r_{i}^{2} \leq u, v \leq r_{i}^{2} .
\end{aligned}
$$

Using Theorem 2.2, we obtain that (3.6) has at least $2 q$ positive 1-periodic solutions $x_{i}, y_{i}$, $1 \leq i \leq q$ with $r_{i}^{1} \leq\left\|x_{i}\right\| \leq r_{i}^{2} \leq\left\|y_{i}\right\|, 1 \leq i \leq q$.

Example 3.2 Consider the differential equation

$$
\left\{\begin{array}{l}
u^{\prime \prime}(t)-u^{\prime}(t)-u=0, \quad t \neq t_{k}, \\
\Delta u\left(t_{k}\right)=u^{2}\left(t_{k}\right), \quad \Delta u^{\prime}\left(t_{k}\right)=-u^{2}\left(t_{k}\right), \quad k \in \mathbb{Z},
\end{array}\right.
$$

where $t_{k}<t_{k+1}$, and there exists $p \in \mathbb{N}$ such that $t_{k+p}=t_{k}+T$.

Taking $m_{1}=\cdots=m_{p}=2, n=1, k_{0}=1, l_{1}=1, l_{2}=2$ and $a=0.7, b=1.7$, one has

$$
\alpha_{k}=-2, \quad \bar{h}(1)=1, \quad \beta_{k_{0}}=-2, \quad g\left(l_{1}\right)=-1,
$$

where $\alpha_{k}, \bar{h}, \beta_{k}, g$ are defined in Corollary 2.1. The conditions $\left(\mathrm{C}_{1}\right)$ and $\left(\mathrm{C}_{2}\right)$ of Corollary 2.1 hold, and hence (3.7) has at least one positive $T$-periodic solution.

If $t_{k}=k,(3.7)$ has a unique positive 1 -periodic solution

$$
u(t)=c_{1} \exp \lambda_{1}(t-k)+c_{2} \exp \lambda_{2}(t-k), \quad k-1<t \leq k,
$$


where

$$
\begin{array}{ll}
\lambda_{1}=\frac{1+\sqrt{5}}{2}, \quad \lambda_{2}=\frac{1-\sqrt{5}}{2}, & A=\frac{\left(1+\lambda_{2}\right)\left(e^{-\lambda_{2}}-1\right)}{\left(1+\lambda_{1}\right)\left(1-e^{-\lambda_{1}}\right)}, \\
c_{2}=\frac{\lambda_{1}-\lambda_{2}}{(1+A)^{2}\left(1+\lambda_{1}\right)}\left(e^{-\lambda_{2}}-1\right), & c_{1}=A c_{2} .
\end{array}
$$

Example 3.3 Consider the differential equation

$$
\left\{\begin{array}{l}
u^{\prime \prime}(t)+\frac{1}{100}(1-u) u=0, \quad t \neq t_{k}, \\
\Delta u\left(t_{k}\right)=\lambda u\left(t_{k}\right), \quad \Delta u^{\prime}\left(t_{k}\right)=-\lambda u\left(t_{k}\right), \quad k \in \mathbb{Z},
\end{array}\right.
$$

where $\lambda>0$ and $t_{k+4}=t_{k}+1$.

We claim that (3.8) has one 1-periodic solution for $\lambda \in(0,0.03)$. In fact, $f(u)=0.01$ (1$u) u, I_{k}(u)=\lambda u, J_{k}(u)=-\lambda u, p=4$. Taking $a=b=1, r=0.1, R=100$, then

$$
\begin{aligned}
& f(u)+u \geq 0, \quad u \in(0,101], \\
& f(u)-\sum_{k=1}^{4} J_{k}\left(v_{k}\right)>0 \quad \text { for } \frac{r}{e} \leq u, v_{1}, \ldots, v_{4} \leq r, \\
& f(u)-\sum_{k=1}^{4} J_{k}\left(v_{k}\right)<\left(1-\frac{R}{e}\right) \frac{1}{e}+400 \lambda<\left(1-\frac{R}{e}\right) \frac{1}{e}+12<0 \quad \text { for } \frac{R}{e} \leq u, v_{1}, \ldots, v_{4} \leq R .
\end{aligned}
$$

By Theorem 2.1, (3.8) has at least one positive 1-periodic solution.

Since

$$
\lim _{u \rightarrow+\infty} \frac{f(u)}{u}=-\infty
$$

there is no constant $\rho>0$ such that $f(u)+\rho^{2} u \geq 0$ for all $u \geq 0$. Hence, the fundamental condition in [12] is not satisfied.

Example 3.4 Consider the differential equation

$$
\left\{\begin{array}{l}
u^{\prime \prime}(t)+C u^{\prime}(t)-u^{\alpha}(t)+\frac{1}{u^{\beta}(t)}=0, \quad t \neq t_{k} \\
\Delta u\left(t_{k}\right)=-\lambda u\left(t_{k}\right), \quad \Delta u^{\prime}\left(t_{k}\right)=-\lambda\left(u^{2}\left(t_{k}\right)+u\left(t_{k}\right)\right)
\end{array}\right.
$$

where $C>1,0<\alpha<1, \beta>0, t_{k}<t_{k+1}, \lambda$ is a positive real parameter, and there exists $p \in \mathbb{N}$ such that $t_{k+p}=t_{k}+T, 1 \leq T \leq C$.

We claim that (3.9) has at least two $T$-periodic solutions if $\lambda>0$ is sufficiently small. In fact, $f(u)=u^{-\beta}-u^{\alpha}, I_{k}(u)=-\lambda u, J_{k}(u)=-\lambda\left(u^{2}+u\right)$. Setting

$$
b=\frac{1}{T}, \quad a=b+C, \quad r_{1}=10^{-4}, \quad r_{2}=100^{\frac{1}{\alpha}}, \quad r_{3}=r_{2} e / \lambda,
$$

we have

$$
f(u)+a b u=u^{-\beta}-u^{\alpha}+a b u \geq u^{-\beta}-u^{\alpha}+u>0 \quad \text { for } u>0,
$$




$$
D_{k}(u)=\lambda(1-b) u+\lambda u^{2} \geq 0 \quad \text { for } u>0 .
$$

\section{Taking}

$$
0<\lambda<\min \left\{\left(\frac{r_{2}^{2-\alpha}}{2 T e^{\alpha}}\right)^{\frac{1}{1-\alpha}}, \frac{T}{2(C+1) p r_{2}^{2}}, \frac{1}{2}\right\}
$$

we have

$$
\begin{aligned}
& T f(u)-\sum_{k=1}^{p}\left[J_{k}\left(v_{k}\right)+(a-b) I_{k}\left(v_{k}\right)\right]>0 \quad \text { for } \frac{r_{1}}{e} \leq u, v_{1}, v_{2}, \ldots, v_{p} \leq r_{1} \text {, } \\
& T f(u)-\sum_{k=1}^{p}\left[J_{k}\left(v_{k}\right)+(a-b) I_{k}\left(v_{k}\right)\right] \\
& \leq T\left(u^{-\beta}-100 / e^{\alpha}\right)+\lambda p\left[r_{2}^{2}+(C+1) r_{2}\right] \\
& \leq T\left(1-100 / e^{\alpha}\right)+\lambda p(C+1)\left(r_{2}^{2}+r_{2}\right) \\
& \leq-T+2(C+1) p \lambda r_{2}^{2}<0 \text { for } \frac{r_{2}}{e} \leq u, v_{1}, v_{2}, \ldots, v_{p} \leq r_{2} \text {, } \\
& T f(u)-\sum_{k=1}^{p}\left[J_{k}\left(v_{k}\right)+(a-b) I_{k}\left(v_{k}\right)\right] \\
& >-T\left(1+\left(r_{3}\right)^{\alpha}\right)+\lambda \frac{r_{3}^{2}}{e^{2}} \\
& \geq-2 T\left(r_{3}\right)^{\alpha}+\frac{r_{2}^{2}}{\lambda}=-2 T \frac{\left(r_{2} e\right)^{\alpha}}{\lambda^{\alpha}}+\frac{r_{2}^{2}}{\lambda}>0 \quad \text { for } \frac{r_{3}}{e} \leq u, v_{1}, v_{2}, \ldots, v_{p} \leq r_{3} \text {. }
\end{aligned}
$$

Hence, (3.8) has at least two $T$-periodic solutions $x_{1}$, $x_{2}$ with $r_{1} \leq\left\|x_{1}\right\| \leq r_{2} \leq\left\|x_{2}\right\| \leq r_{3}$.

\section{Competing interests}

The authors declare that they have no competing interests.

\section{Authors' contributions}

Authors typed, read and approved the final draft.

\section{Author details}

'Department of Mathematics, Hunan University of Science and Technology, Xiangtan, Hunan 411201, P.R. China.

${ }^{2}$ Department of Mathematics, Hunan First Normal College, Changsha, Hunan 410205, P.R. China.

\section{Acknowledgements}

The authors wish to express their thanks to the referee for his/her very valuable suggestions and careful corrections. The work is supported by the NNSF of China (11171085), Hunan Provincial Natural Science Foundation of China (2015JJ2068) and Scientific Research Fund of Hunan Provincial Education Department (14A028).

Received: 15 April 2015 Accepted: 6 September 2015 Published online: 28 September 2015

\section{References}

1. Bainov, DD, Simeonov, PS: Impulsive Differential Equations: Periodic Solutions and Applications. Longman, Harlow (1993)

2. Bainov, DD, Hristova, SG, Hu, S, Lakshmikantham, V: Periodic boundary-value problem for systems of first-order impulsive differential equations. Differ. Integral Equ. 2, 37-43 (1989)

3. Chen, J, Tisdell, CC, Yuan, R: On the solvability of periodic boundary value problems with impulse. J. Math. Anal. Appl. 331, 902-912 (2007)

4. Chu, J, Nieto, JJ: Impulsive periodic solutions of first-order singular differential equations. Bull. Lond. Math. Soc. 40 143-150 (2008)

5. Erbe, LH, Liu, X: Existence results for boundary value problems of second order impulsive differential equations. J. Math. Anal. Appl. 149(1), 56-69 (1990) 
6. Heidarkhani, S, Ferrara, M, Salari, A: Infinitely many periodic solutions for a class of perturbed second-order differential equations with impulses. Acta Appl. Math. (2014). doi:10.1007/s10440-014-9970-4

7. Luo, Z, Nieto, J: New results for the periodic boundary value problem for impulsive integro-differential equations. Nonlinear Anal. TMA 70, 2248-2260 (2009)

8. Qian, $D, L i, X$ : Periodic solutions for ordinary differential equations with sublinear impulsive effects. J. Math. Anal. Appl. $303,288-303(2005)$

9. Sun, J, Chen, H, Nieto, JJ, Otero-Novoa, M: Multiplicity of solutions for perturbed second-order Hamiltonian systems with impulsive effects. Nonlinear Anal. TMA 72, 4575-4586 (2010)

10. Zhang, H, Li, Z: Periodic and homoclinic solutions generated by impulses. Nonlinear Anal., Real World Appl. 12, $39-51$ (2011)

11. Zhang, $X, L i, X$, Jiang, D, Wang, K: Multiplicity positive solutions to periodic problems for first order impulsive differential equations. Comput. Math. Appl. 52, 953-966 (2006)

12. Shen, J, Wang, W: Positive periodic solutions for second order impulsive differential equations. Demonstr. Math. XLIV(2), 297-316 (2011)

13. Zhou, Q, Jiang, D, Tian, Y: Multiplicity of positive solutions to period boundary value problems for second order impulsive differential equations. Acta Math. Sci. 26, 113-124 (2010)

14. Guo, D, Lakshmikantham, V: Nonlinear Problems in Abstract Cones. Academic Press, Orlando (1988)

\section{Submit your manuscript to a SpringerOpen ${ }^{\odot}$ journal and benefit from:}

- Convenient online submission

- Rigorous peer review

- Immediate publication on acceptance

- Open access: articles freely available online

- High visibility within the field

- Retaining the copyright to your article 